\title{
Effect of nicotine dependence on quality of life and sleep quality in patients with lung cancer who continue to smoke after diagnosis
}

\author{
Fen $\mathrm{Gu}^{1}$, Xue-Fei $\mathrm{Li}^{1}$, Jin-Fu Xu${ }^{2}$, Guang-Hui Gao ${ }^{1}$, Yi-Fan $\mathrm{Wu}^{1}$, Cai-Cun Zhou ${ }^{1}$ \\ ${ }^{1}$ Oncology Department, ${ }^{2}$ Respiratory Department, Shanghai Pulmonary Hospital, Tongji University School of Medicine, Shanghai 200433, China \\ Contributions: (I) Conception and design: F Gu, CC Zhou; (II) Administrative support: All authors; (III) Provision of study materials or patients: All \\ authors; (IV) Collection and assembly of data: YF Wu; (V) Data analysis and interpretation: XF Li; (VI) Manuscript writing: All authors; (VII) Final \\ approval of manuscript: All authors. \\ Correspondence to: Cai-Cun Zhou, MD, PhD. Oncology Department, Shanghai Pulmonary Hospital, Tongji University School of Medicine, Shanghai \\ 200433, China. Email: Caicunzhoudr@163.com.
}

\begin{abstract}
Background: Hundreds of millions of Chinese individuals continue to smoke and rates of lung cancer still continue to rise. However, there were few studies that examined the effects of nicotine dependence on quality of life (QOL) and sleep quality in lung cancer patients. This study aimed to investigate the effect of nicotine dependence on QOL and sleep quality in lung cancer patients who continue to smoke after diagnosis.

Methods: This cross-sectional survey study included 202 patients with lung cancer. Smokers were separated into two groups based on the Fagerstrom Test for Nicotine dependence: the low dependence (LD) ( $<4$ score) group ( $n=59$ ) and the high dependence (HD) ( $\geq 4$ score) group ( $n=143)$. Both Chinese version of the European Organization for Research and Treatment of Cancer Quality of Life Core Questionnaire 30 (EORTC QLQ-C30) and Chinese version of Pittsburgh Sleep Quality Index (PSQI) were used to evaluate the two groups of lung cancer patients. Then we analyzed the difference of QOL and sleep quality between two distinct nicotine dependence groups.

Results: Physical functioning, role functioning, emotional functioning, cognitive functioning, global health status and social functioning items in the LD group were significantly higher than the HD group $(\mathrm{P}<0.001)$. Fatigue, nausea/vomiting, pain, dyspnea, insomnia, appetite loss, diarrhea and financial problems in the LD group were significantly lower than those in the HD group $(\mathrm{P}<0.001)$. Significantly higher scores in the HD group were found concerning the three sleep components including sleep duration, sleep efficiency and daytime function. The mean global PSQI score in the HD group was significantly higher than the LD group $(\mathrm{P}=0.014)$.
\end{abstract}

Conclusions: These findings suggest that lung cancer patients who continue to smoke after diagnosis should receive health education in order to improve their QOL and quality of sleep after the word education. This can be useful for clinicians and nurses who are trying to motivate smokers to quit smoking.

Keywords: Nicotine dependence; quality of life (QOL); sleep quality; lung cancer; smoke

Submitted Aug 15, 2017. Accepted for publication Dec 28, 2017.

doi: $10.21037 /$ jtd.2018.05.12

View this article at: http://dx.doi.org/10.21037/jtd.2018.05.12

\section{Introduction}

According to the 2014 Surgeon General's Report, smoking by cancer patients and survivors causes adverse outcomes including increased overall mortality, increased causespecific mortality, and increased risk for second primary cancers (1). But hundreds of millions of Chinese patients continue to smoke and rates of diagnosis of lung cancer are also gradually increasing. National registry data on lung cancer in China do not yet cover even a fifth of the national population (2). People who smoke are more likely to suffer from squamous cell lung carcinoma and small cell 
lung cancer (2). Taghizadeh et al. investigated 504 lung cancer patients and reported that $13(2.6 \%)$ patients were never-smoker, 302 (59.9\%) stopped smoking more than 3 months prior, while the remaining 189 (37.5\%) were current-smoker (3). History of cigarette smoking has been associated with decreased overall survival among patients who received systemic treatment after diagnosis (4).

Health-related quality of life (QOL) has become an extremely important aspect for patients receiving systemic treatment, especially when the treatment intent was supportive or palliative or when the survival benefits are modest. Many studies have reported the impact of smoking on QOL of patients with lung cancer $(5,6)$, but the QOL of patients with lung cancer who still continue to smoke is less concerned. Sleep disturbance is also a common problem in lung cancer patients. $M$. Nishiura et al reported that $56 \%$ of lung cancer patients had sleep disturbances (7). A study conducted in multiethnic sample of US adults demonstrated that current smokers and current smokeless tobacco users had twice the odds of insufficient sleep compared to non-smokers/non-smokeless tobacco users (8). However, few studies have examined the effect of nicotine dependence on QOL and sleep quality in patients with lung cancer.

Hence, this study was conducted to investigate the effect of nicotine dependence on lung cancer and sleep quality in patients with lung cancer who still continue to smoke after initial diagnosis.

\section{Methods}

Two hundred and four patients with lung cancer from the Shanghai Pulmonary Hospital were included in this quantitative study from November 2016 to February 2017. The patients' age ranged from 37 to 83 years old and their mean age were $61.46 \pm 8.41$ years old. The participants were first hospitalized patients, current smokers and were diagnosed using accepted diagnostic criteria based on clinical assessment, molecular analyses of tumor biopsy specimens and computed tomography of the chest. Participants with impaired cognitive function, other secondary tumors, heart, cerebral or renal failure, those who lost follow-up or without sufficient clinical data and had ceased cigarette smoking were excluded from this study. The outcomes were assessed before receiving any treatments. There were 204 individuals sampled and 202 individuals responded (response rate, $99.02 \%)$. Informed consent was obtained from participants in the study.

\section{Questionnaires and definitions}

Demographic information regarding gender, age, marital status, education level, annual household income, employment, diagnosis, stage of disease were recorded. Smoking status included years of regular smoking, that is, the age when the participant first started smoking and current cigarettes smoked per day.

We employed the Fagerstrom Test for Nicotine Dependence (FTND) (9) to assess the physical nicotine dependence. The FTND consists of 6 items that evaluate the quantity of cigarettes smoked, diurnal patterns of use (time to first cigarette upon awakening, smoking more during the first $2 \mathrm{~h}$ of the day, the cigarette one would hate most to give up), and impaired control over use (smoking when ill, difficulty refraining from smoking when prohibited). Total scores range from 0 to 10 . We classified nicotine dependence into two categories, low dependence (LD; FTND <4) and high dependence (HD; FTND $\geq 4)(10)$. Cronbach internal consistency coefficients of FTND items for participants $\alpha=0.75$ (11).

QOL was measured by EORTC QLQ-C30 Version 3.0 (12). The 30-item questionnaire of EORTC QLQ-C30 assesses five functional domains (i.e., physical, role, emotional, cognitive and social function) which include eight cancer-related symptoms, financial difficulty and global health status. Each item is rated on a scale from 1 (not at all) to 4 (very much). All scales/items are transformed to scores ranging from 0 to 100 . Higher scores on the five functional subscales and global health status represent better QOL. Lower scores on the eight symptom items and financial difficulty represent better QOL. The EORTC QLQ-C30 was identified as one of the best instruments to measure QOL in lung cancer patients (13). In this study, we used the Chinese version of the EORTC QLQ-C30.

Sleep disturbance was assessed by Chinese version of Pittsburgh Sleep Quality Index (PSQI). The PSQI is a 19item self-report questionnaire which assesses the types and frequency of sleep disturbances experienced over the last month (14). It consists of 5 peer rating questions, and 19-items with self-assessment questions, including seven components which are subjective sleep quality, sleep latency, sleep duration, habitual sleep efficiency, sleep disturbance, use of sleep medication and daytime dysfunction. Each component ranges from 0 (no difficult) to 3 (very difficult). The sum of scores of these seven components yields one global score, which ranges from 0 to 21 . Higher scores represent poorer sleep (15). Score 
of more than one component indicated existence of sleep problems in this component (16). According to the study of Wang et al., the test-retest reliability of the PSQI is 0.994 . The split-half reliability coefficient of the PSQI was 0.824 , and the overall Cronbach's alpha coefficient was 0.845 (17). It means that the PSQI has good reliability and validity.

\section{Statistical methods}

The characteristics of patients in the LD and HD groups were compared using a two sample $t$-test (or Wilcoxon rank sum test) for continuous variables and a Chi-square test (or Fisher's exact test) for categorical variables. The difference of duration of smoking cessation was performed using nonparametric test. When the data was in a normal distribution, the number was described as mean \pm standard deviation; when the data was skewed, the number was described as median (quartile range).

Comparison of the mean scores of EORTC QLQ-C30 functioning scales were conducted by student's $t$-test, with a two-sided significance level of 0.05 . The scores of EORTC QLQ-C30 symptom scales displayed skew distribution. Hence, the differences in the symptom scales (fatigue, nausea/vomiting, pain, dyspnea, insomnia, appetite loss, diarrhea and constipation) and financial problems by nicotine dependence were conducted by Wilcoxon-MannWhitney test.

Comparison of the mean scores of the PSQI was conducted by student's $t$-test with a two sided significance level of 0.05. But sleep duration, habitual sleep efficiency, use of sleep medication and day time dysfunction were skewed distribution. The nicotine dependence differences were evaluated by using Wilcoxon-Mann-Whitney test.

Data analysis was performed using Statistical Package for Social Science (SPSS) version 20.0 (IBM Inc., Chicago Illinois, USA, 2011).

\section{Results}

A total of 202 patients were enrolled, and the demographic characteristics of the study population were presented in Table 1. The mean age was $61.5 \pm 8.4$ years, and all the patients were male. There were no significant differences in the demographic parameters including age, smoking years, age at initiation, quit smoking frequency, duration of smoking cessation, household income, employed, education level, lung cancer type and cancer stage between the LD group and the HD group. However, compared with the LD group, the number of cigarette per day and the fagerstrom score were significant higher in the HD group $(30.06 \pm 14.11$ vs. $18.70 \pm 9.75,6.08 \pm 1.65$ vs. $1.61 \pm 1.15$, both $\mathrm{P}<0.001$, respectively). The percentages of single patients were also higher in the HD group (Table 1).

\section{QOL by nicotine dependence}

Patients in the LD group demonstrated the significant higher scores of global health status and EORTC QLQ-C30 functioning scales scores including physical functioning, emotional functioning, and cognitive functioning (all $\mathrm{P}<0.05)$ (Table 2), indicating a higher level of QOL.

In addition, the $\mathrm{LD}$ group patients also reported statistically significant better financial problems and EORTC QLQ-C30 symptom scales scores including fatigue, nausea/vomiting, pain, dyspnea, insomnia, appetite loss, diarrhea, except constipation (Table 2).

\section{Sleep quality by nicotine dependence}

The patients in the HD group had significantly worse sleep quality with higher the PSQI scores $(\mathrm{P}<0.05)$ (Table 3). Among seven components of sleep quality, they were significantly worse in sleep duration, habitual sleep efficiency and sleep disturbance (Table 3).

\section{Discussion}

To our knowledge, this is the one of the meaningful studies to examine the effect of nicotine dependence on QOL and sleep quality in lung cancer patients who continues to smoke after diagnosis. The QOL and sleep quality in patients with lung cancer who continue to smoke after diagnosis have not been adequately studies. In this cross-sectional study, we found that: (I) lung cancer patients who continue to smoke after diagnosis with lower FTND had higher QOL than patients with higher FTND; (II) compared with patients with higher FTND, lung cancer patients who continue to smoke after diagnosis with lower FTND had better sleep quality. Our study has demonstrated for the first time the profile of sleep quality in patients with lung cancer who continue to smoke after diagnosis.

The current study confirmed the results of previous studies that light smokers reported best QOL profile, while heavy smokers reported worst QOL $(4,18,19)$. Serious effects of QOL and morbidity were associated with 
Table 1 Characteristics of 202 lung cancer patients by nicotine dependence

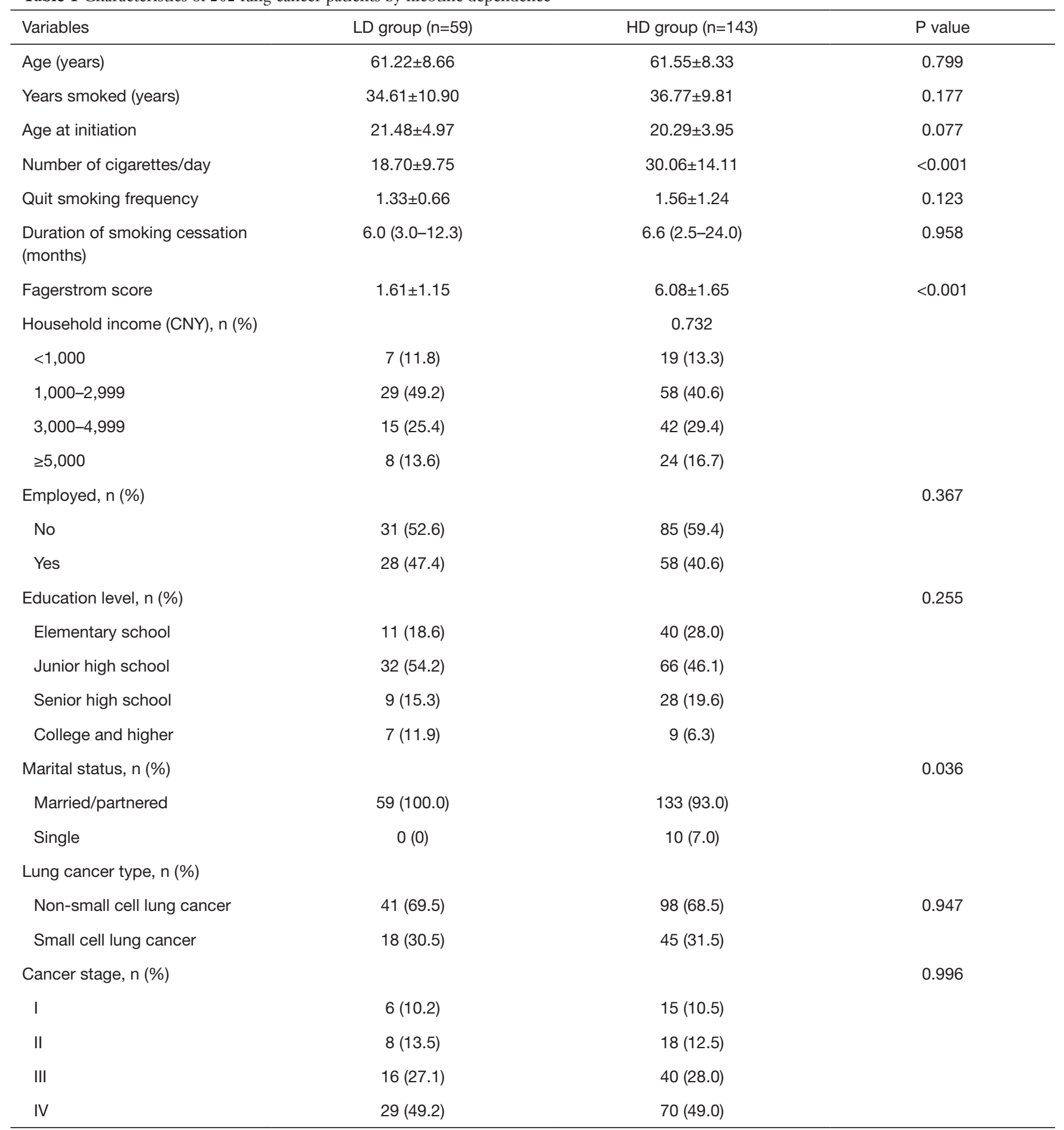

Data in parentheses are N (\%) or mean \pm SD or median (quartile range); LD group, the low dependence group (FTND $<4$ ); HD group, the high dependence group (FTND $\geq 4$ ); duration of smoking cessation (months): the number of months during the patient's life since they began cigarette smoking when they had temporarily quit. FTND, Fagerstrom Test for Nicotine Dependence. 
Table 2 The QOL of 202 lung cancer patients by nicotine dependence

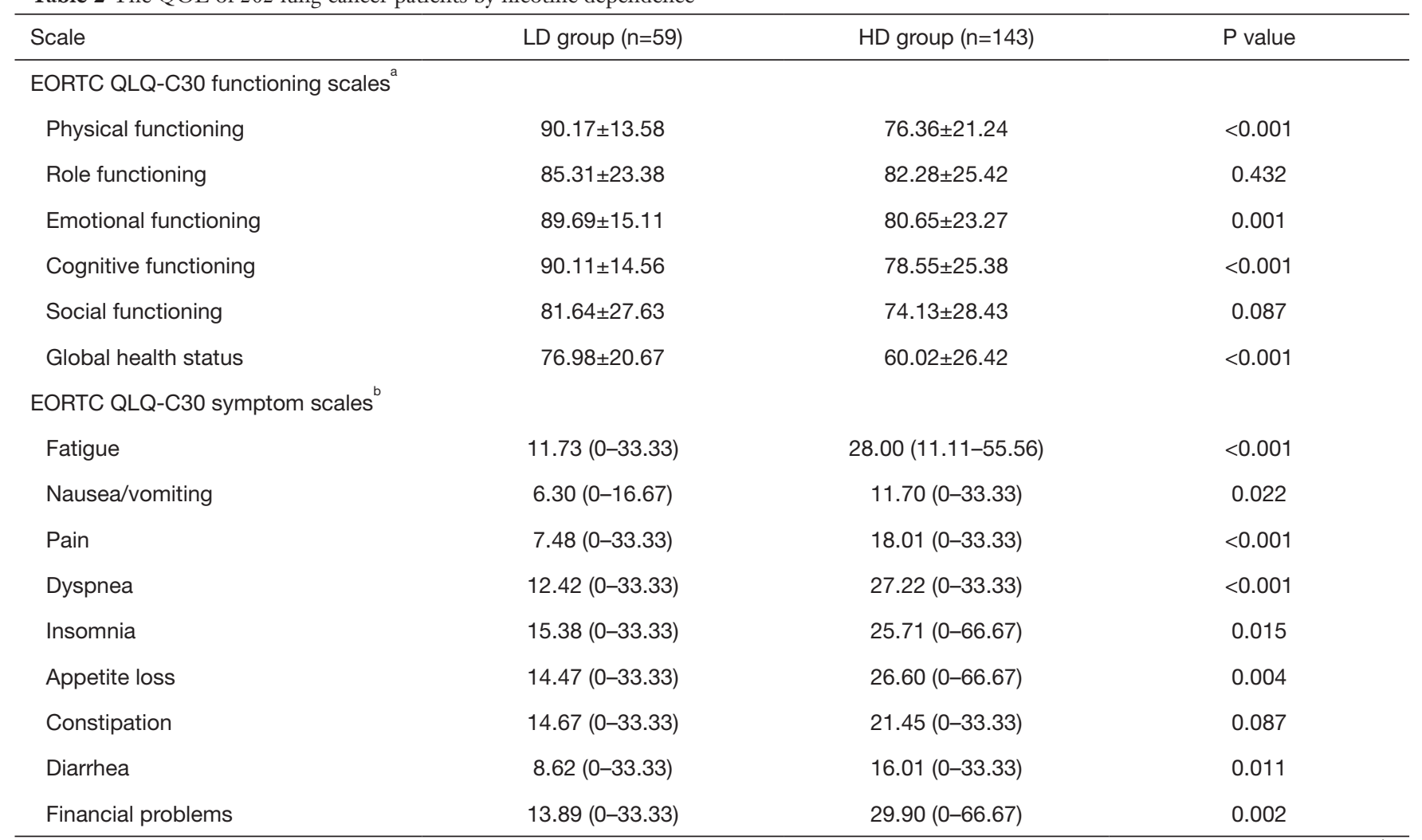

Data in parentheses are mean \pm SD or median (quartile range). ${ }^{a}$, higher scores indicate better functioning and global health status; ${ }^{b}$, higher scores indicate more symptoms. LD group, the low dependence group (FTND <4); HD group, the high dependence group (FTND 24); QLQ-C30: the European Organization for Research and Treatment of Cancer quality-of-life core questionnaire. QOL, quality of life; LD, low dependence; FTND, Fagerstrom Test for Nicotine Dependence; HD, high dependence.

Table 3 The sleep quality of 202 lung cancer patients by nicotine dependence

\begin{tabular}{lccc}
\hline Factors & LD group $(\mathrm{n}=59)$ & HD group $(\mathrm{n}=143)$ & $\mathrm{P}$ value \\
\hline Subjective sleep quality & $0.88 \pm 0.59$ & $1.01 \pm 0.65$ & 0.175 \\
Sleep latency & $1.18 \pm 0.75$ & $1.24 \pm 0.96$ & 0.658 \\
Sleep duration & $0(0-1)$ & $1(0-2)$ & 0.002 \\
Habitual sleep efficiency & $0(0-1)$ & $0(0-2)$ & 0.030 \\
Sleep disturbance & $1.14 \pm 0.51$ & $1.36 \pm 0.67$ & 0.009 \\
Use of sleep medication & $0(0-1)$ & $0(0-0)$ & 0.724 \\
Daytime dysfunction & $0(0-1)$ & $0(0-1)$ & 0.108 \\
PSQl scores & $5.00 \pm 3.21$ & $6.31 \pm 3.48$ & 0.014 \\
\hline
\end{tabular}

Data in parentheses are mean \pm SD or median (quartile range). LD group, the low dependence group (FTND $<4$ ); HD group, the high dependence group (FTND $\geq 4$ ). LD, low dependence; HD, high dependence; PSQI, The Pittsburgh Sleep Quality Index; FTND, Fagerstrom Test for Nicotine Dependence. 
smoking problem (4). Different from these above results, we studied the QOL and sleep quality profile in lung cancer population who continued to smoke after diagnosis. These results challenged the belief that patients with cancer usually stop smoking after diagnosis or appearing symptoms (20). In this study, the differences of QOL existed in patients with different FTND. These statistical differences provide important implications for avenues to smoking cessation both in a community and a hospital. In addition, our study found that the most important factors affecting QOL included physical functioning, emotional functioning, global health status and cognitive functioning. The symptoms of fatigue, nausea/vomiting, pain, dyspnea, insomnia, appetite loss and diarrhea were more obvious in lung cancer patients who continued to smoke after diagnosis.

Sleep disturbances were more common in the advanced lung cancer patients and had a negative impact on patients QOL (21). Several researches have reported that cigarette smoking might impair memory and sleep quality in healthy population $(22,23)$. But few researches concentrated mainly on the effects of cigarette smoking on sleep quality of lung cancer patients. Cigarette smoking behavior and poor sleep quality of lung cancer patients might share common biological or psychological grounds. Mak et al. found that adolescent smoking was associated with snoring and difficulty breathing during sleep (24). In a multicenter sample of US adults, compared to nonsmokers/non- smokeless tobacco users, those who were both current smokers and current smokeless tobacco users had twice the odds of insufficient sleep (8). Different from above research results, our study had demonstrated for the first time the profile of sleep quality in patients with lung cancer who continue to smoke after diagnosis. In this study, lung cancer patients with higher FTND had higher PSQI scores, indicating poor sleep quality. Our research revealed that sleep duration, habitual sleep efficiency and sleep duration were most closely affected in lung cancer patients with high nicotine dependence after diagnosis. This study raised the importance of addressing smoking cessation in lung cancer patients who continued to smoke after diagnosis.

The current study is limited by its cross-sectional design. A longitudinal study would be necessary to evaluate the effect of nicotine dependence on evolution and survival outcome of lung cancer who continues to smoke after diagnosis.

\section{Conclusions}

Our study confirmed that the QOL and sleep quality were worse in lung cancer patients with high nicotine dependence after diagnosis than those with low nicotine dependence. We should concentrate on the global health status, physical functioning, emotional functioning, cognitive functioning, some symptoms and financial problems of patients with high nicotine dependence. We should also be concerned about the sleep duration, habitual sleep efficiency and sleep disturbance of the lung cancer patients with high nicotine dependence. This study can be useful for clinicians and nurses who are trying to motivate smokers to quit smoking.

\section{Acknowledgements}

The authors thank Rong Jiang for her help to revise the article and also thank Ying Yu, Hui-Ying Zhu and Li-Hua Huang for their help with the data collection.

Funding: This study was funded by the project of Shanghai municipal health and family planning commission (No. 201640327) and the talent project of Shanghai pulmonary hospital (No. HR2016001).

\section{Footnote}

Conflicts of Interest: The authors have no conflicts of interest to declare.

Ethical Statement: The ethics committee of the Shanghai Pulmonary Hospital, China approved the study protocol for this retrospective analysis (No. k17-121).

\section{References}

1. Dobson Amato KA, Hyland A, Reed R, et al. Tobacco Cessation May Improve Lung Cancer Patient Survival. J Thorac Oncol 2015;10:1014-9.

2. Stone ECA, Zhou C. Slowing the Titanic: Chinaacco Cessation May Improve Lung. J Thorac Oncol 2016;11:2053-65.

3. Taghizadeh N, Taylor KL, MacEachern P, et al. Tobacco use and motivation to stop smoking among long-term smokers who are ineligible for lung cancer screening. Lung Cancer 2017;111:101-7.

4. Chen J, Qi Y, Wampfler JA, et al. Effect of cigarette smoking on quality of life in small cell lung cancer patients. 
Eur J Cancer 2012;48:1593-601.

5. Ferketich AK, Otterson GA, King M, et al. A pilot test of a combined tobacco dependence treatment and lung cancer screening program. Lung Cancer 2012;76:211-5.

6. van der Aalst CM, de Koning HJ. Biochemical verification of the self-reported smoking status of screened male smokers of the Dutch-Belgian randomized controlled lung cancer screening trial. Lung Cancer 2016;94:96-101.

7. Nishiura M, Tamura A, Nagai H, et al. Assessment of sleep disturbance in lung cancer patients: Relationship between sleep disturbance and pain, fatigue, quality of life, and psychological distress. Palliat Support Care 2015;13:575-81.

8. Sabanayagam C, Shankar A. The association between active smoking, smokeless tobacco, second-hand smoke exposure and insufficient sleep. Sleep Med 2011;12:7-11.

9. Fagerström KO. Measuring degree of physical dependence to tobacco smoking with reference to individualization of treatment. Addict Behav 1978;3:235-41.

10. Rios-Bedoya CF, Snedecor SM, Pomerleau CS, et al. Association of withdrawal features with nicotine dependence as measured by the Fagerstrom Test for Nicotine Dependence (FTND). Addict Behav 2008;33:1086-9.

11. Prochaska JJ, Leek DN, Hall SE, et al. Cognitive interviews for measurement evaluation of the Fagerstrom Test for Nicotine Dependence (FTND) in smokers with schizophrenia spectrum disorders. Addict Behav 2007;32:793-802.

12. Quinten C, Coens C, Ghislain I, et al. The effects of age on health-related quality of life in cancer populations: A pooled analysis of randomized controlled trials using the European Organisation for Research and Treatment of Cancer (EORTC) QLQ-C30 involving 6024 cancer patients. Eur J Cancer 2015;51:2808-19.

13. Chen ML, Yu CT, Yang CH. Sleep disturbances and quality of life in lung cancer patients undergoing chemotherapy. Lung Cancer 2008;62:391-400.

14. Hinz A, Glaesmer H, Brähler E, et al. Sleep quality in the general population: psychometric properties

Cite this article as: Gu F, Li XF, Xu JF, Gao GH, Wu YF, Zhou CC. Effect of nicotine dependence on quality of life and sleep quality in patients with lung cancer who continue to smoke after diagnosis. J Thorac Dis 2018;10(5):2583-2589. doi: 10.21037/jtd.2018.05.12 of the pittsburgh sleep quality index, derived from a german community sample of 9284 people. Sleep Med 2017;30:57-63.

15. Passos MH, Silva HA, Pitangui AC, et al. Reliability and validity of the Brazilian version of the Pittsburgh Sleep Quality Index in adolescents. J Pediatr (Rio J) 2017;93:200-6.

16. Mollayeva T, Thurairajah P, Burton K, et al. The Pittsburgh sleep quality index as a screening tool for sleep dysfunction in clinical and non-clinical samples: A systematic review and meta-analysis. Sleep Med Rev 2016;25:52-73.

17. Wang G, Wang F, Li Q, et al. Positive correlation between cerebrospinal fluid glutamate levels and Pittsburgh Sleep Quality Index scores in northern Chinese subjects. Sleep Med 2016;23:123-4.

18. Cooley ME, Sarna L, Kotlerman J, et al. Smoking cessation is challenging even for patients recovering from lung cancer surgery with curative intent. Lung Cancer 2009;66:218-25.

19. Wilson D, Parsons J, Wakefield M. The Health-Related Quality-of-Life of Never Smokers, Ex-smokers, and Light, Moderate, and Heavy Smokers. Prev Med 1999;29:139-44.

20. Conlon K, Pattinson L, Hutton D. Attitudes of oncology healthcare practitioners towards smoking cessation: A systematic review of the facilitators, barriers and recommendations for delivery of advice and support to cancer patients. Radiography 2017;23:256-63.

21. Lou VW, Chen EJ, Jian H, et al. Respiratory Symptoms, Sleep, and Quality of Life in Patients With Advanced Lung Cancer. J Pain Symptom Manage 2017;53:250-6.e1.

22. Liu JT, Lee IH, Wang CH, et al. Cigarette smoking might impair memory and sleep quality. J Formos Med Assoc 2013;112:287-90.

23. Jaehne A, Unbehaun T, Feige B, et al. How smoking affects sleep: a polysomnographical analysis. Sleep Med 2012;13:1286-92.

24. Mak KK, Ho SY, Thomas GN, et al. Smoking and sleep disorders in Chinese adolescents. Sleep Med 2010;11:268-73. 